\title{
The Effect of Prooxidant Diets on Some Experimental Mycobacterial Infections ${ }^{\star}$
}

\author{
VIENY BERGEL \\ Director, Laboratorio de Investigcciones Leprologicass, Rosarrio, Argentina \\ (Former Guest Inestigator of the Rockefeller Institute, New York, U.S.A.).
}

\section{INTRODUCTION}

In an extensive series of experiments Negre ${ }^{1}$, Dubós ${ }^{2}$, Hedgecock ${ }^{3}$ and others established a correlation between lipid metabolism and the virulence of mycobacteria.

The administration of the prooxidant diets to mice and the effects on subsequent mycobacterial infections is the subject of this report.

In a general way, prooxidant diets are those which can produce in a living organism a state of prooxidation, i.e., the biological condition in which undesirable autocatylitic oxidations occur with formation of hydroperoxides in the tissues. To bring this about there must be 2 dietetic pre-requisites: $(a)$ the lack or a low concentration of vitamin $\mathrm{E}$ or any other biological antioxidant in the diet, and $(b)$ a great quantity of unsaturated fatty acids wiuh high carbon number.

Experiments conducted by Bergel ${ }^{4}$ and Mason et $a l{ }^{5}$ demonstrated that Mycobacterium leprae can grow and reproduce actively in rats fed prooxidant diets. These investigations were conducted to determine if such dietary factors influenced other mycobacterial infections.

MATERIAL AND METHODS

Bacterial Cultures

The experiments were carried out with the following strains of bacteria:-

Mycobacterium tuberculosis Vallee: a bovine strain maintained in a state of high virulence for white mice by monthly passage in Tween-albumin medium.

BCG (Phipps): a strain of BCG from the Henry Phipps Institute of Philadelphia (U.S.A.). The cultures were grown at $37^{\circ} \mathrm{C}$. in Tween-albumin medium and were used when 10 to 12 days old.
Mycobacterium Fortuitum strain Penso: a rapid growth mycobacterium. The cultures were grown at $37^{\circ} \mathrm{C}$. in Dubós-Tween medium and were used when 2 days old.

Staphylococcus aureus strain Giorgio: a coagulase positive strain used in the form of 18-hour old cultures in beef heart infusion-peptone broth.

Klebsiella pneumoniae type $\mathrm{C}$ : used in the form of an 18-hour old culture in beef heart infusion-peptone broth.

I.PS: a highly purified preparation of lipopolysaccharide prepared from the typhoid bacillus.

\section{Animals}

The mice used were males, litter mates from the Rockefeller Institute Swiss Strain. They were raised at the Rockefeller Institute, weaned at 3 weeks of age and taken immediately after weaning. They were housed in metal cages on grids without bedding. The mice were weighed in groups of 5 at the beginning of the experiment and at regular intervals thereafter until the time of infection. Animals were infected after they had been on the experimental diets for a variable period, and by different routes, as it is indicated in each case.

\section{Diets}

Pellets: From Purina Laboratory Chow, St. Louis, Mo., U.S.A., with the following composition:-

\footnotetext{
* Work done at Rockefeller Institute for Medical Research, New York, U.S.A., under a Research Grant of Ciba Laboratories of Summit, N.J., U.S.A.
} 
Crude Protein (min.) . 2 23.0\%

Crude Fat (min.) $\quad$. $\quad 5.0 \%$

Crude Fibre $(\max$.$) \quad . \quad 6.0 \%$

Special Diets: Two different types of prooxidant diets were used: a cod liver oil diet and a linseed oil diet. The basic dry mix was prepared as follows:-

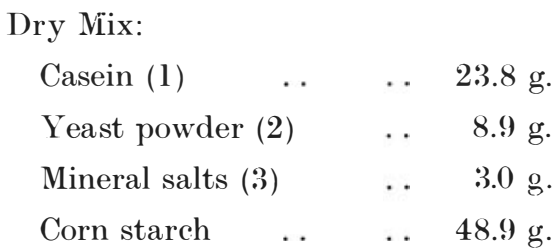

The cod liver oil (4) diet and the linseed oil (5) diet were prepared by adding $15 \%$, of the oils - in weight - to the basic dry mix.

(1) Vitamin E free casein from Nutritional Biochemical Corporation, Cleveland, O. U.S.A.

(2) Pure dry yeast (brewers' type) from Standard Brand Incorporated, New York, N.Y., U.S.A.

(3) Salt mixture H.M.W. from Nutritional Biochemical Corporation, Cleveland, O., U.S.A.

(4) Raw cod liver oil from E. R. Squibb and Sons, New York, N.Y., U.S.A.

(5) Raw linseed oil from Deusch Boy Inc.' New York, N.Y., U.S.A.

All food remnants were removed daily and cages cleaned once weekly. Tap water was administered ad lib. to all animals.

\section{Validity of the results}

The experiments were carried out with groups of 10 to 20 mice and the survival times between groups recorded but no attempt was made to assess the findings in statistical terms. Instead many experiments were repeated several times to underline the results.

Because of the large number of animals used autopsies were not carried out in all the cases to confirm the cause of death: they were made only in special cases as indicated later.
RESULTS

\section{Nutritional Value of the prooxidant diet}

In order to determine the nutritional affect of the prooxidant diet-with linseed oil -2 groups of 14 mice were placed in individual cages from the age of $18-21$ days. One group received pellets and the other the prooxidant diet with linseed oil. Mice were weighed every 3 days and their weight was recorded up to a period of 37 days. Fig. 1 shows the average weight of mice from each group: they are almost the same.

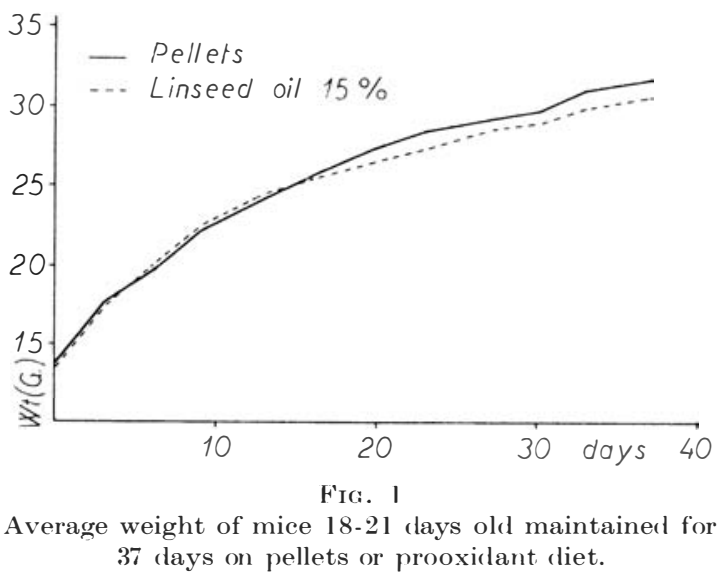

It is important to point this out since the prooxidant diet is quite a complete one with a high content of protein suitable for keeping the animals in a very good nutritional condition. Its only undesirable effect is an increased autooxidation in the tissues of animals on this diet.

\section{Inoculation of Mycobacterium tuberculosis Vallee to mice with prooxidant diet}

In 4 different experiments 18-21 days old mice in groups of $15-20$ were placed on the following 3 diets: $(a)$ pellets; $(b)$ the prooxidant diet with linseed oil; and $(c)$ the prooxidant diet with cod liver oil. After a period of 14, 29 and 43 days on diet in the 4 experiments, all the mice were inoculated intravenously in the caudal vein with $0.05 \mathrm{ml}$. of Vallee Tween Albumin IV in $0.2 \mathrm{ml}$. of saline. Figs. 2, 3, 4 and 5 illustrate the mortality that occurs in the selected groups of mice. 


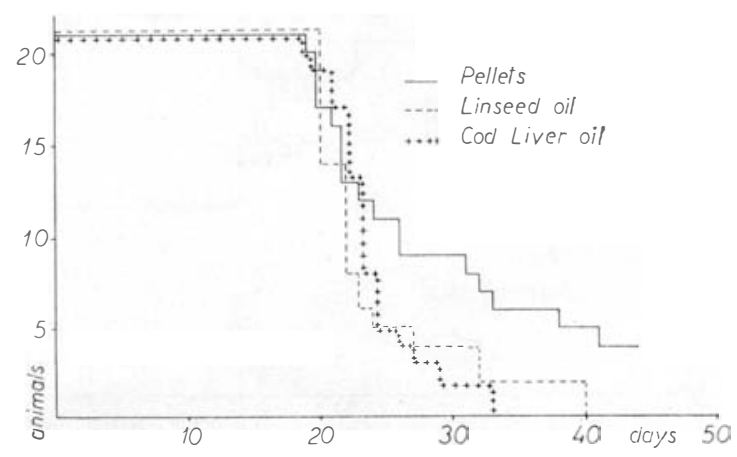

FI(x. 2

Effect of the prooxidant diet on the mortality of mice inoculated with $M$. tuberculosis Vallee after 14 days on diet. Ordinates indicate number of mice surviving at different periods of time after infection (abscissae).

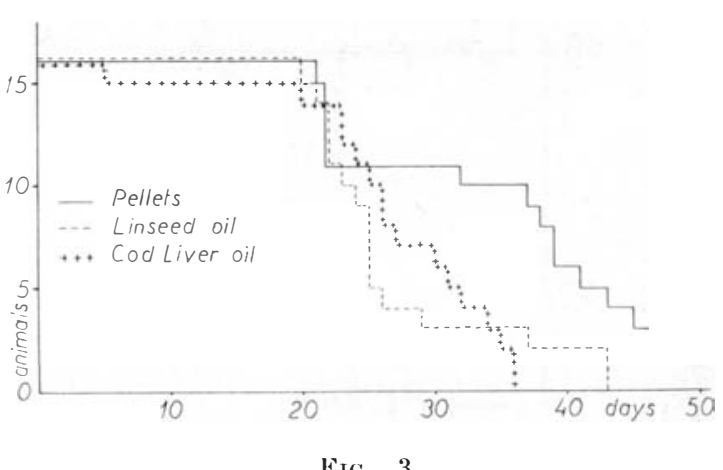

Effect of the prooxidant diet on the mortality of mice inoculated with M. tuberculosis Vallee after 29 days on diet. Ordinates indicate number of mice surviving at different periods of time after infection (abscissae).
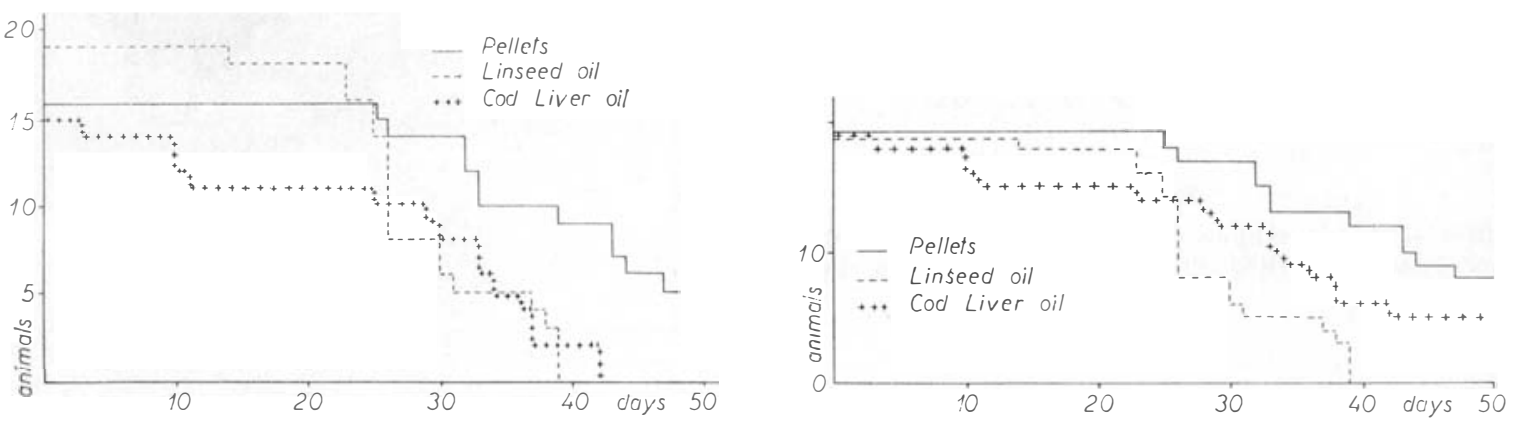

Fig. 4 and Fir. 5

Effect of the prooxidant diet on the mortality of mice inoculated with $M$. tuberculosis Vallee after 43 days on diet. Ordinates indicate number of mice surviving at different periods of time after infection (abscissae).

Results are consistent for the 4 experiments described, showing that animals receiving a prooxidant diet are less resistant to experimental tuberculosis infection produced by inoculation of bovine tubercle. The differences observed between the 2 prooxidant diets used-with linseed oil and cod liver oil-seem to have little significance.

\section{Inoculation of BCG Phipps to mice with prooxidant diet}

Groups of 15 white mice 18-21 days old were placed on the 3 diets: $(a)$ pellets; $(b)$ prooxidant diet with linseed oil; and $(c)$ prooxidant diet with cod liver oil. After 14 days on the diet all animals were inoculated intravenously with $0.2 \mathrm{ml}$. of a culture of BCG Phipps. Fig. 6 shows the results of this experiment.

The results of this experiment show that the prooxidant diet of cod liver oil increases the death rate due to intravenous injection of BCG in mice. The surviving mice, sacrificed 75 days after inoculation, showed a greater number of lesions in the lungs of animals on prooxidant diet than in control animals though the lesions were not so intense as to cause death. 


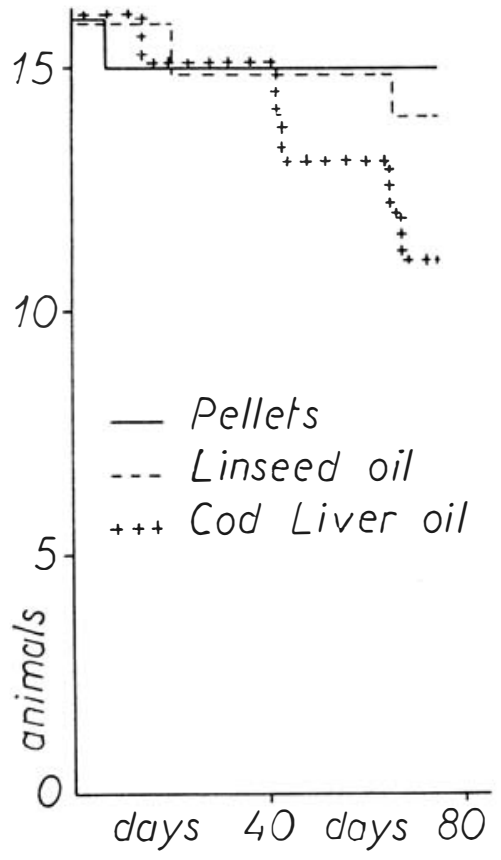

FIG. 6

Effect of the prooxidant diet on the mortality of mice inoculated with BCG Phipps after 14 days on diet.

\section{Inoculation of Mycobacterium fortuitum Penso} to mice with prooxidant diet

In 2 different experiments groups of 20 white mice 18-21 days old were placed on 2 diets: $(a)$ pellets, and $(b)$ prooxidant diet with linseed oil. In one experiment after 21 days on diet and in the other after 36 days on diet all animals were inoculated intravenously with $0.2 \mathrm{ml}$. of a 2 days old culture of $M$. fortuitum Penso. Figs. 7 and 8 show the results of these 2 experiments.

These 2 experiments show that the prooxidant diet evidently increased the death rates due to intraveous inoculation of $M$. fortuitum Penso in mice. It must be remarked that the average weight of the mice at the time of inoculation was $27 \mathrm{~g}$. for the control group receiving pellets, and $32.5 \mathrm{~g}$. for the animals receiving linseed oil.

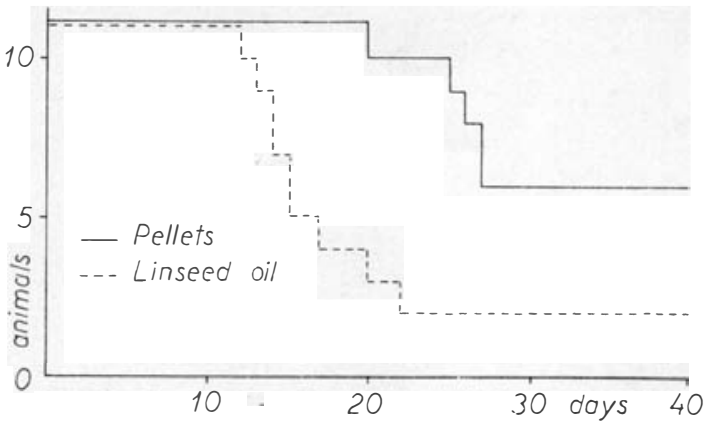

FIG. 7

Effect of the prooxidant diet on the mortality of mice inoculated with $M$. fortuiturn Penso after 21 days on diet. Ordinates indicate number of mice surving at different period of time after infection (abscissae).

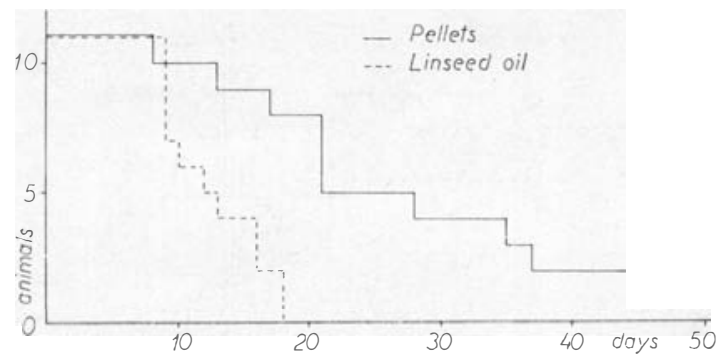

FIG. 8

Effect of the prooxidant diet on the mortality of mice inoculated with $M$. fortuitum Penso after 36 days on diet. Ordinates indicate number of mice surviving at different periods of time after infection (abscissae).

\section{Inoculation of Staphylococcus aureus (Giorgio)} to mice with prooxidant diet

White mice 18-21 days old were placed on the following 2 diets: $(a)$ pellets, and $(b)$ prooxidant diet with linseed oil. After 22 days on these diets all animals were inoculated intravenously with $0.05 \mathrm{ml}$. of an 18-hour old culture of Staphylococcus aureus (Giorgio). Likewise 2 other groups of animals on the same dietary regimen were inoculated intraperitoneally with $0.1 \mathrm{ml}$. of the same culture plus $5 \mathrm{mcg}$. of LPS (lipopolysaccharide) and the other group with $0.1 \mathrm{ml}$. of the Giorgio culture plus $10 \mathrm{meg}$. of LPS. Figs. 9, 10 and 11 show the results of these experiments. 


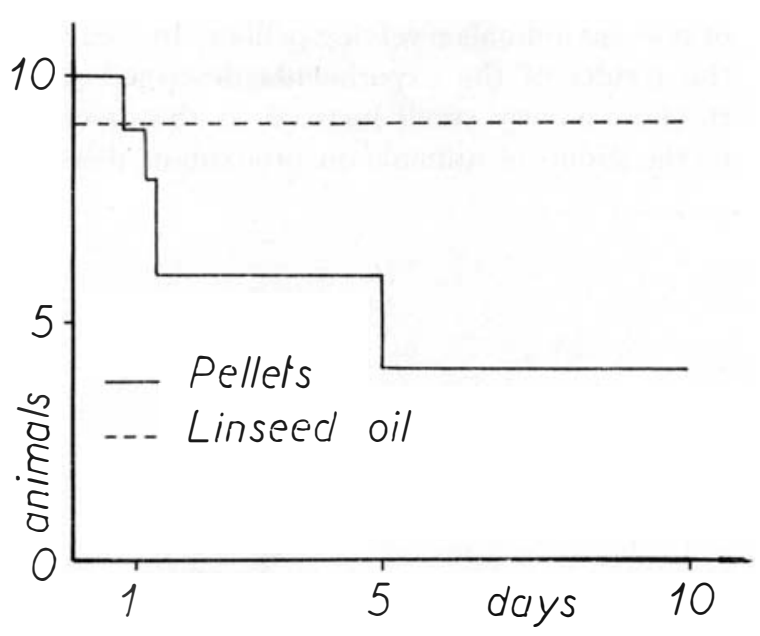

FIG. 9

Effect of the prooxidant diet on the mortality of mice inoculated with Staphylococcus aureus (Giorgio) after 22 days on diet. Ordinates indicate number of mice surviving at different periods of time after infection (abscissae).

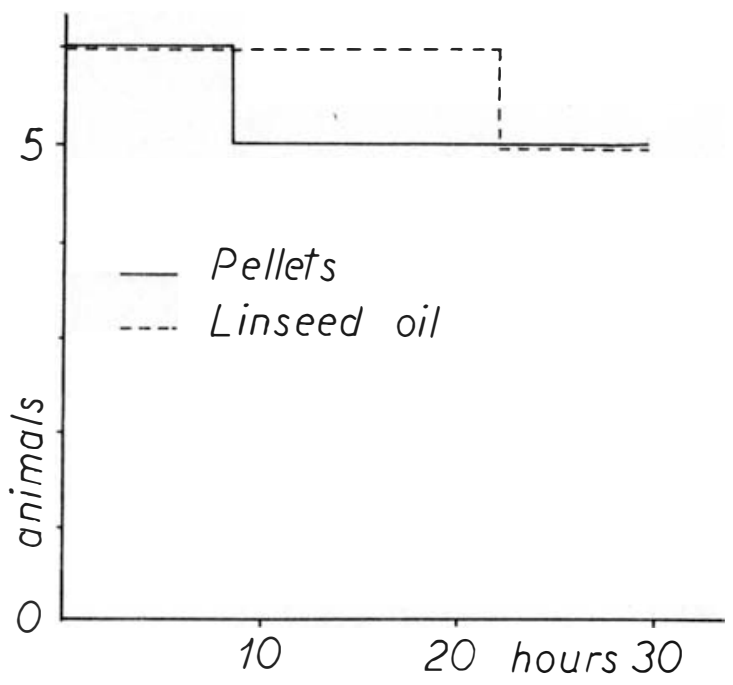

FIG. 11

Effect of the prooxidant diet on the mortality of mice inoculated with Staphylococcus aureus (Giorgio) plus LPS (10 gammas) after 22 days on diet; intraperitoneally in $0.2 \mathrm{ml}$. Ordinates indicate number of mice surviving at different period of time after infection (abscissae).

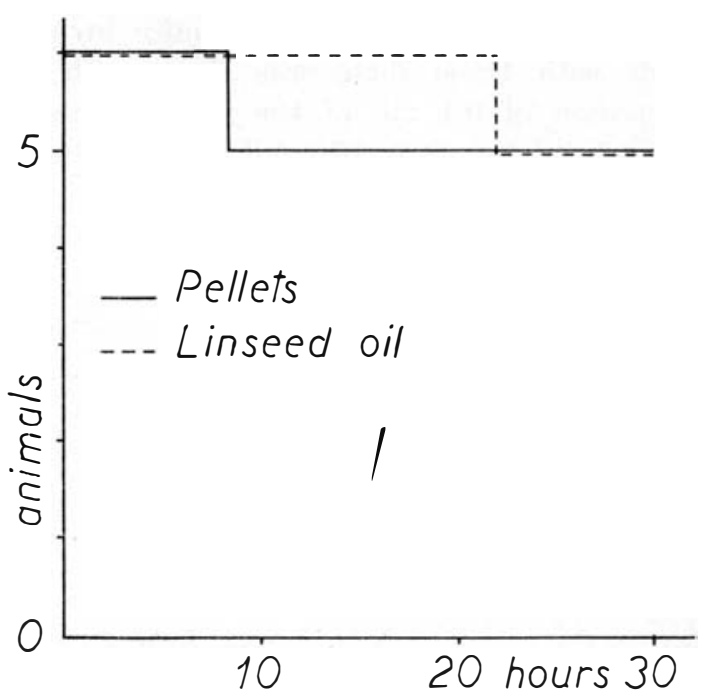

FIG. 10

Effect of the prooxidant diet on the mortality of mice inoculated with Staphylococcus aureus (Giorgio) plus LPS (5 gammas) after 22 days on diet; intraperitoneally in $0.2 \mathrm{ml}$. Ordinates indicate indicate of mice surviving at different period of time after infection (abscissae).

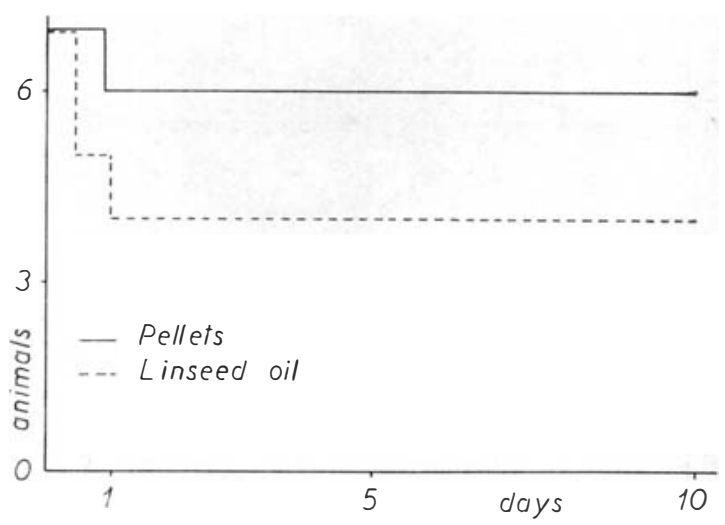

FIG. 12

Effect of the prooxidant diet on the mortality of mice inoculated with Staphylococcus aureus (Giorgio) plus LPS intraperitoneally. Ordinates indicate number of mice surviving at different period of time af ter infection (abscissae). 
One other experiment with 6 mice in each group with these diets was done with an inoculation of $0.1 \mathrm{ml}$. of the Giorgio culture plus $0.1 \mathrm{ml}$. of $1 / 20$ LPS all injected intraperitoneally. Results are shown in Fig. 12.

These experiments show that the animals on prooxidant diets are as resistant to inoculation of Staphylococcus aureus - whether LPS is added or not-as animals receiving pellets. In contrast, the results of the experiments described seem to show a very small increase in the defences of the group of animals on prooxidant diets.

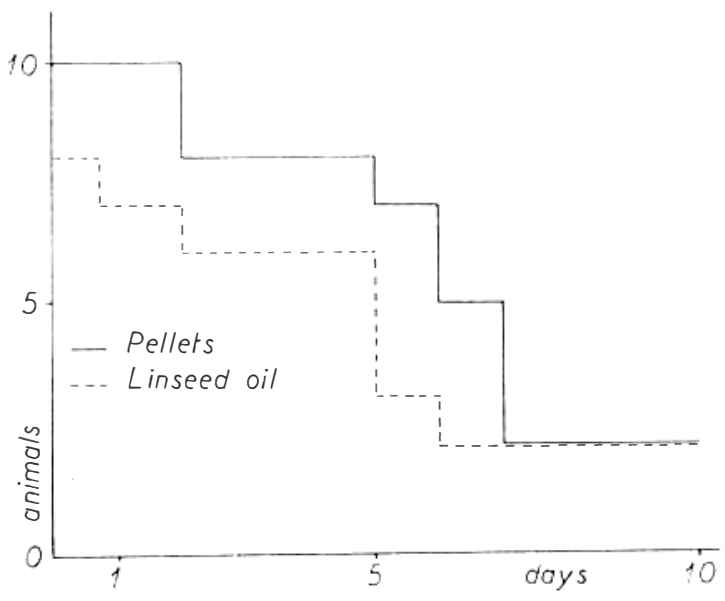

FIG. 13

Effect of the prooxidant diet on the mortality of mice inoculated with Klebsiella pneumoniae (Friedländer) after 42 days on diet. Ordinates indicate number of mice surviving at different period of time after infection (abscissae).

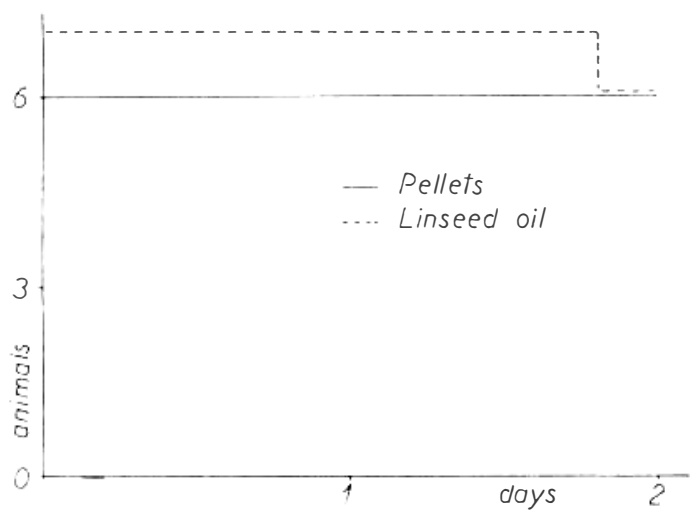

Fig. 14

Effect of the prooxidant diet on the mortality of mice inoculated with L,PS $(200 \mathrm{mcg}$. in $0.2 \mathrm{ml}$. intraperitoneally) after 42 days on diet. Ordinates indicate number of mice surviving at different periods of time after inoculation (abscissae).

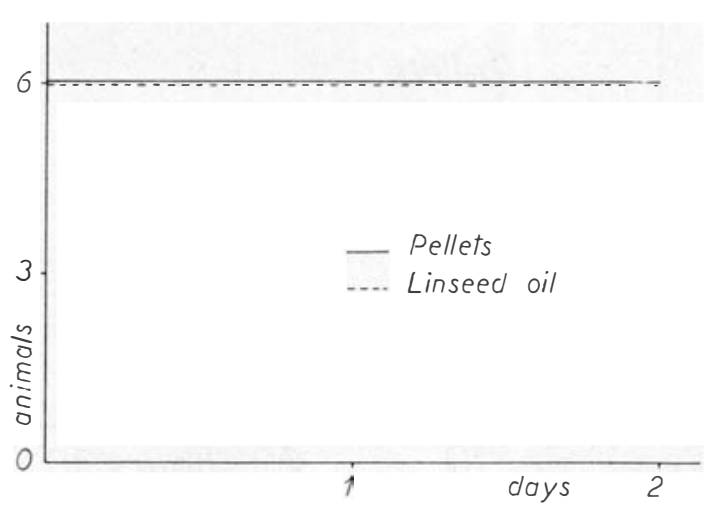

Hig. I5

Effect of the prooxidant diet on the mortality of mice inoculated with LPS $(400 \mathrm{meg}$. in $0.2 \mathrm{ml}$. intraperitoneally) after 42 days on diet. Ordinates indicate number of mice surviving at different periods of time af ter inoculation (abscissae). 
6. Inoculation of Klebsiella pneumoniae (Friedländer) to mice with prooxidant diet

Groups of 10 white mice 18-21 days old were placed on the 2 following diets: $(a)$ pellets, and (b) prooxidant diet with linseed oil. After 42 days on these diets all animals were inoculated intravenously with $0.2 \mathrm{ml}$. of $1 / 20$ of the mentioned culture of Friedländer. Fig. 13 shows the mortality in both groups of animals.

This experiment reveals similar susceptibility to pneumococcal infection in animals under prooxidant diets and animals receiving pellets.

\section{Inoculation of Lipopolysaccharide (LPS) to mice with prooxidant diet}

In 2 different experiments groups of 6 mice 18-21 days old were placed on the following 2 diets: $(a)$ pellets, and $(b)$ prooxidant diet with linseed oil. After 42 days on diet all the animals were inoculated in the following manner: one group with $200 \mathrm{mcg}$. of LPS in $0.2 \mathrm{ml}$. intraperitoneally; and the other group with $400 \mathrm{meg}$. in the same way. Figs. 14 and 15 show the comparative mortality in both experiments.

From the results obtained we can say that both types of diet give similar defence against inoculation of LPS in mice.

\section{DISCUSSION}

From the above-mentioned experiments it can be concluded that the prooxidant diet, high in unsaturated fatty acids and low in vitamin $\mathrm{E}$, increased the susceptibility of mice to experimental infections with Mycobacterium tuberculosis Vallee, BCG Phipps and Mycobacterium fortuitum Penso. However, animals fed these prooxidant diets are as resistant to staphylococcal and Friedländer infections and to the LPS as animals fed pellets. It should be pointed out that animals gain weight equally well with all the diets described, namely the prooxidant and the standard ones.
These results are in agreement with the work of Furuta ${ }^{6}$ which demonstrates that rodents fed a prooxidant diet become more susceptible to Mycobacterium lepraemurium (Stefansky) infection; and also with the already quoted works of Bergel and Mason in which it is concluded that Mycobacterium le prae can grow when inoculated into rats fed a prooxidant diet.

From all these experiments we can conclude that prooxidant diets have a specific action on infections due to mycobacteria.

\section{SUMMARY}

Mice fed prooxidant diets, with cod liver oil or linseed oil, gain weight just as well as those fed with standard diets.

Mice fed with the prooxidant diets, high in unsaturated fatty acids and low in vitamin $\mathrm{E}$, become more susceptible to infections produced by the inoculation of Mycobacterium tuberculosis Vallee, BCG Phipps and Mycobacterium fortuitum Penso.

Mice fed prooxidant diets are as resistant to staphylococcal, Friedländer and lipopolysaccharide infections as animals which are fed pellets.

\section{ACKNOWLEDGEMENT'S}

The author expresses his gratitude to Dr. René J. Dubós and Russell W. Schaedler under whose valuable guidance the present work was done.

\section{BIBLIOGRAPHY}

1. Negre, L. Ann. de l'Inst. Pasteur, 1932, 49, 319.

2. Dubos, R. J. .J. of Experimental Med., 1955, $101,59$.

3. Hedgecock, L. W. Proc. Soc. Expt. Biol. and Med., $1948,68,141$.

4. BERGEL, M. Lep. Rev., 1959, 30, 159.

5. MASON, K. E. and MEI, Y. D. Symposium on Research in Leprosy. Baltimore, 1961, p. 264.

6. Furuta, m. Communication to 33rd National Congress of Leprosy, Japan, 6 April, 1960. 